\title{
A 4 year ERCP Profile of Tertiary Care Hospital: Indications and Diagnosis
}

\author{
AFTAB HAIDER ALVI ${ }^{1}$, AIJAZ ZEESHAN KHAN CHACHAR ${ }^{2}$, RAHEEL PARVAIZ ${ }^{3}$ \\ ${ }^{1}$ Associate Professor of Gastroenterology and Hepatology, Fatima Memorial Hospital, Shadman, Lahore \\ ${ }^{23}$ Senior Registrar Medicine, Fatima Memorial Hospital, Shadman, Lahore \\ 3PGR Nephrology Fatima Memorial Hospital, Lahore \\ 3Correspondence to Dr. Aijaz Zeeshan Khan Chachar Email: dr_aijaz84@hotmail.com Cell:0333-2612096
}

\begin{abstract}
Aims: To find the indications and diagnosed conditions in the cases reported in Fatima Memorial Hospital Lahore. Also, the prevalence of this procedure amongst the genders in our setting. To establish statistical association between indications and diagnosis.

Methodology: All the ERCP procedures done in 4 years from January, 2011 to January 2014 were compiled. The data was gathered using a simple form after the intervention. A total of 554 patients' data was available, all participants were above the age of 18 years. A response rate $93 \%$ was recorded for who consented and allowed to share their information anonymously in the study. Chi square was used to find statistical association, with $p$-value $<0.005$ was taken as significant. IBM SPSS 22 was used for data input and evaluation.

Results: In our study $35 \%$ of the participants were males and $65 \%$ were females. $58.33 \%$ of the patients underwent sphincterotomy. The highest number of patient's indicated of undergoing ERCP was $58.33 \%$ because of obstructive jaundice without any cause on further investigations. Choledocholithiasis was the commonest diagnosis with $36.6 \%$. A strong association between the indications and the final diagnosis was also found with $p$-value of 0.000 . The success rate of the intervention was $79 \%$ making it a safer procedure to be applied.

Conclusion: More females have undergone this procedure. Obstructive jaundice was declared as major indication with choledocholithiasis as the commonest condition diagnosed. It was a successful procedure in our setting.

Keywords: ERCP, Gastroenterology, Intervention
\end{abstract}

\section{INTRODUCTION}

Endoscopic Retrograde Cholangiopancreatography (ERCP) is a diagnostic as well as a therapeutic procedure combining endoscopy and fluoroscopy to evaluate as well as treat the diseases of biliary and pancreatic ducts. The deep enteroscopic ERCP and laparascopic ERCP have been devised to answer the complex anatomy, although the success rates for enteroscopic-assisted ERCP stayed as $63 \%$ in trials reported ${ }^{1}$. The major risks involve reaction to perforation, bleeding and a major cause of pancreatitis $^{2}$. Pancreatitis due to ERCP is the commonest complication of the procedure with hemorrhage as the second one, $3.5 \%$ and $1.3 \%$ respectively ${ }^{3}$. The diagnostic ERCP reports a total of $0.2 \%$ mortality although it gets doubled in therapeutic ERCP procedure ${ }^{4,5}$. Perforation statistics range up to 0.1 to $0.6 \%$ making it a lesser common complication, although none of these meta-analysis numbers are promising enough to establish new scientific facts upon it 5 .

Received on 14-02-2021

Accepted on 27-05-2021
With magnetic resonance pancreatography (MRCP), ERCP has almost been replaced for diagnostic purposes although the only intent now is for a therapeutic intervention. ERCP is still popularly practiced and performed quite safely. ERCP is a popular procedure but is not free of the complications that are associated during and after the procedure. As a whole, it is just not the procedure but the expertise of the endoscopists, the trained team and the interventional technique can change the outcome. Appropriate patient selection, anesthesia and the available technology can affect the aspects of this technique. If complications occur, prompt response can alter the mortality numbers ${ }^{6,7}$.

The study was carried in department of gastroenterology, Fatima Memorial Hospital with 594 patients in a span of 4 years. This cross-section of 4 years accounted the various indications, the diagnosis reported and various other particulars of the patient and the procedure.

\section{METHODS AND MATERIAL}

A cross-sectional study was carried out in Fatima Memorial Hospital, a tertiary care hospital in the 
Lahore city. The span of the study ranged in 4 years, starting from late year 2011 to year 2015. A pre designed performa including the age, gender, major complaints, type of anesthesia, status of sphincterotomy, probable diagnosis and therapeutic intervention all was noted down.

The total sample size of the study was 554 which were taken by the convenience, as all the procedures carried in the department were included in the study. For the ease of data handling, every consultant was asked to report the patient data separately and then collectively pooled for data analysis. All the study participants were above the age of 18 , although the inclusion was irrespective of the presenting complaint. All the participants gave written consent for the use of their data and the recorded response rate was $93 \%$. The information collected was entered in statistical software IBM SPSS 22 for evaluation and analysis. For all categorical variables such as age, gender, indications, anesthesia application and diagnosis were reported in frequencies. Statistical test of chi square was applied in finding the significance between the variables, considering $p$-value $<0.005$ as significant.

\section{RESULTS}

A total of 554 people took part in our study, out of which $35 \%$ (194 out of 554) were males and 65\% (360 out of 554 ) were females. The mean age the study participants were $53.49 \pm 15.86$ years old. Majority of the procedures, $34.5 \%$ were carried in year 2013 and least number was reported in year 2011, as low as 4.3\%. In year 2012 and 2014, the percentages were $33.8 \%$ and $27.4 \%$ respectively.

The data gathered from the study also showed the frequency of sphincterotomy done during the procedure. Out of 554, the data for sphincterotomy was entered for 540 patients $(97.47 \%)$. Majority of the patients $58.33 \%$ ( 315 out of 540 ) had undergone sphincterotomy. Another important data contribution of this study was the application of anesthesia. Data for 543 patients was added regarding anesthesia, out of which $53.41 \%$ (290 out of 543 ) were given anesthesia and $46.59 \%$ (253 out of 543 ) were not given. No pain-induced mortality was reported.

Regarding the indications reported in our department, data for 7 patients was incomplete; a total of 547 patient's data was entered. $46.6 \%$ (258 out of 547) underwent ERCP because of obstructive jaundice without any cause in further investigations, making it the leading cause of ERCP in our investigation. Second was common bile duct stone $30.5 \%$ (169 out of 547). Least reported indication was intrahepatic cholangiocarcinoma, which was seen in $1.1 \%$ (6 out of 547$)$

$36.6 \%$ (203 out of 554) were diagnosed with choledocholithiasis, making it the most diagnosed condition in our 4 year cross-section. 26.9\% (149 out of 554) were diagnosed with cholangiocarcinoma, making it the second most common condition. Choledochocyst and dilated CBD were the least diagnosed cases, with $0.4 \%$ each (2 out of 554 ).

A test of significance, chi square was applied to find the statistical significance between the indications and the final diagnosis of the cases. A significant association was established with $p$-value of 0.000 , degree of freedom at 66 and Pearson chi square value of 828.198 . Overall, $79 \%$ (437 out of 554 ) were successful procedures, although $21 \%$ (117 out of 554) were reported unsuccessful.

Table 1: Frequency table of indications of ERCP amongst the study participants of our study in 4 years.

\begin{tabular}{|l|c|c|}
\hline Indication & n & \%age \\
\hline Common Bile Duct Stone & 169 & 30.5 \\
\hline Extra-hepatic cholangiocarcinoma & 14 & 2.5 \\
\hline Intrahepatic hepatic cholangiocarcinoma & 6 & 1.1 \\
\hline Peri-ampullary Tumor & 34 & 6.1 \\
\hline CBD Sludge & 45 & 8.1 \\
\hline CBD Injury/ leak & 21 & 3.8 \\
\hline $\begin{array}{l}\text { Obstructive jaundice without any further } \\
\text { investigations }\end{array}$ & 258 & 46.6 \\
\hline Total & 547 & 98.7 \\
\hline
\end{tabular}

Table 2. The cross tabulation of the indications and the diagnoses made from the investigation. The chi square was applied in this tabulation, with a p-value of 0.000

\begin{tabular}{|c|c|c|c|c|c|c|c|c|}
\hline & \multicolumn{7}{|c|}{ Indications } & \multirow[t]{2}{*}{ Total } \\
\hline & $\begin{array}{l}\text { CBD }^{\mathrm{a}} \\
\text { Stone }\end{array}$ & $\begin{array}{c}\text { EH } \\
\text { Cho.Cab }\end{array}$ & $\begin{array}{c}\text { IH } \\
\text { Cho.Ca }\end{array}$ & PAT $^{c}$ & $\begin{array}{l}\text { CBD } \\
\text { Sludge }\end{array}$ & $\begin{array}{c}\text { CBD } \\
\text { Injury/ } \\
\text { leak }\end{array}$ & $\begin{array}{c}O^{\mathrm{d}} \text { without } \\
\text { any cause in } \\
\text { investigations }\end{array}$ & \\
\hline choledocholithiasis & 136 & 0 & 0 & 0 & 29 & 0 & 37 & 202 \\
\hline CA gall bladder & 1 & 1 & 0 & 0 & 0 & 0 & 5 & 7 \\
\hline choledochocyst & 0 & 0 & 0 & 0 & 0 & 0 & 2 & 2 \\
\hline peri-ampulary tumor & 0 & 0 & 0 & 27 & 1 & 0 & 6 & 34 \\
\hline CA of head of pancreas & 0 & 1 & 0 & 1 & 0 & 0 & 20 & 22 \\
\hline stent replacement & 5 & 0 & 0 & 2 & 4 & 1 & 11 & 23 \\
\hline $\begin{array}{l}\text { Obstructive jaundice due to unknown } \\
\text { reason }\end{array}$ & 3 & 0 & 0 & 0 & 1 & 0 & 40 & 44 \\
\hline cholangiocarcinoma & 17 & 12 & 6 & 3 & 5 & 1 & 101 & 145 \\
\hline
\end{tabular}




\begin{tabular}{|c|c|c|c|c|c|c|c|c|}
\hline dilated CBD on USG & 0 & 0 & 0 & 0 & 2 & 0 & 0 & 2 \\
\hline Itrogenic CBD injury & 6 & 0 & 0 & 0 & 1 & 19 & 20 & 46 \\
\hline stent removal & 1 & 0 & 0 & 0 & 0 & 0 & 3 & 4 \\
\hline blocked CBD stent & 0 & 0 & 0 & 1 & 2 & 0 & 13 & 16 \\
\hline Total & 169 & 14 & 6 & 34 & 45 & 21 & 258 & 547 \\
\hline
\end{tabular}

\section{DISCUSSION}

Our study documented some important frequencies amongst our population. Firstly, the extensive difference amongst the gender distribution of the ERCP procedure shows that it is more carried in females than males. This can be a possibility that the associated symptoms that lead to ERCP are needed more common in females than males. Majority of our study participants underwent the sphincterotomy which shows the high demand of it amongst the ERCP patients, probably more evaluation and studies are needed to establish it an essential component for the protocol of this procedure. The frequency of obstructive jaundice without any further investigation shows a two-fold picture; one that adequate work-ups are needed to diagnose it or probably it was the reluctance of the consultants to proceed to the sophisticated tests to save the money of the patients. It is also a possibility that medical science has to yet probe in to overcome the limitations linked in it. The most common diagnosed case was choledocholithiasis which brings out to investigate the etiological factors prevalent in our population as well as the dietary intake that has recently been shifted with advent of fast foods.

Aawsaj from UK, brought CBD exploration versus laparoscopic ERCP, the collected data expanded at the period of 15 years at NHS Foundation Trust. The ambiguities were addressed sufficiently where laparoscopic ERCP was stated as a safe procedure in emergency as well as in routine. The investigators suggested a transcystic approach. This study was also satisfactory because the followup period was of a mean with 6 months and the major complications were noted as well. Majority of the patients had stone clearance, three died during the course of their follow-up, two had other medical complications and only one had abdominal sepsis. This study established that laparoscopic approach may become a major protocol, which may require more expertise can be the alternative to answer the complications $^{8}$.

ERCP as a sophisticated procedure and it always require proficiency of the gastroenterologist but it is also necessary for the attending to see if there is a need for other specialties to collaborate or not. With a prior reconstructive surgeries or removal of parts of gastrointestinal tract may change the general procedure into a more complicated one. This is where interventional radiologists, imaging consultants and surgical team is needed. The different anatomy because of enteral anastomosis made because of removal of a certain part may bring life threatening complication during the intervention. For promising results, every consultant should reevaluate and if needed should make it a multidisciplinary approach for the ease of the patient ${ }^{9}$.

As like other procedures, ERCP isn't always the perfect fit. It is also associated with complications and the range from mild to moderate is never an absolute status for ERCP. As the study mentioned before stating it a safer procedure, yet has complexities. A prospective study covering more than 16000 patients from ERCP brought down almost 1000 patients suffering from various complications with 55 fatalities. The co-morbidities of these complicated patients are not itemized although we can assume the limitations in the management of such huge sample. Complications included infections, pancreatitis, bleeding and perforations. Cardiovascular and anesthesia related complications were also noted but comparatively in lesser numbers. This study concluded with high number of morbidity and mortality as compared to older studies and statistical analysis ${ }^{10}$. In contrast to this, another study testifying ERCP an entirely a safe procedure and adding that it is the perfect solution for diagnosing primary sclerosing cholangitis and other disease of biliary system with less complications ${ }^{11}$. This can come to a standard where benefits versus risks are pooled together and the one with more sum becomes the choice.

The study carried by us could have been more elaborative if appropriate follow up was done and instead of descriptive data, analysis pertaining to symptomatology and other details on first visit and subsequent visits were also carried. The data could have also been compared with other similar setting to see the differences. The choice of ERCP and the patient comparison amongst the consultants could also bring newer questions in motion. Idea is that a continuous surveillance is needed on such interventions, where the risks are still debatable. An extensive cohort is needed amongst different cases, symptoms and even history of the patients. Moreover, the health watchdogs must issue a 
standardized patient selection protocol or a grading system to help the physicians in selecting appropriate candidates for it. For this widespread data is required to establish such facts. Further probing is required in this chapter.

\section{CONCLUSION}

ERCP is a successful procedure, involving sphincterotomy in majority of the cases. Obstructive jaundice is the most commonly reported indication for ERCP. This brings us to carry the procedure in such setting. Also, by our study population, choledocholothiasis is commonest diagnosed condition. The setting in which we carried the study had no extensive life impairments or mortalities concluding ERCP to be a safe intervention for diagnosing and treating the patient.

\section{REFERENCES}

1. Kedia P, Sharaiha RZ, Kumta NA, Kahaleh M. Internal EUSdirected transgastric ERCP (EDGE): game over. Gastroenterology. 2014 Sep; 147(3):566-8.

2. http://www.nlm.nih.gov/medlineplus/ency/article/007479.htm

3. Andriulli A, Loperfido S, Napolitano G, et al. Incidence rates of post-ERCP complications: a systematic survey of prospective studies. Am J Gastroenterol 2007;102:1781-8
4. Freeman ML, Nelson DB, Sherman S, et al. Complications of endoscopic biliary sphincterotomy. N Engl J Med 1996;335: 909-18.

5. Loperfido S, Angelini G, Benedetti G, et al. Major early complications from diagnostic and therapeutic ERCP: a prospective multicenter study. GastrointestEndosc 1998;48:110

6. Quality improvement of gastrointestinal endoscopy: guidelines for clinical application. GastrointestEndosc 1999;49:842-4. 91

7. Hasan AG, Brown WR. A model for mortality-morbidity conferences in gastroenterology. GastrointestEndosc 2008;67:515-8.

8. Aawsaj Y, Light D, Horgan L. Laparoscopic common bile duct exploration: 15-year experience in a district general hospital. Surg Endosc. 2015 Aug 26.[Epub ahead of print] PubMed PMID: 26307600

9. Amer S, Horsley-Silva JL, Menias CO, Pannala R. Endoscopic retrograde cholangiopancreatography in patients with surgically altered gastrointestinal anatomy. Abdom Imaging. 2015 Aug 25. [Epub ahead of print] PubMed PMID: 26304586.

10. Andriulli A, Loperfido S, Napolitano G, Niro G, Valvano MR, Spirito F, Pilotto A, Forlano R. Incidence rates of post-ERCP complications: a systematic survey of prospective studies. Am J Gastroenterol. 2007 Aug;102(8):1781-8.

11. van den Hazel SJ, Wolfhagen $E H$, van Buuren $H R$, van de Meeberg PC, Van Leeuwen DJ. Prospective risk assessment of endoscopic retrograde cholangiography in patients with primary sclerosing cholangitis. Dutch PSC Study Group. Endoscopy. $2000 \quad$ Oct;32(10):779-82. 